\title{
Eficacia y seguridad del tratamiento empírico con piperacilina/tazobactan como monoterapia en episodios de neutropenia y fiebre en niños con cáncer: revisión sistemática y meta-análisis
}

\author{
Efficacy and safety of empirical treatment with piperacillin/tazobactan as monotherapy \\ in episodes of neutropenia and fever in children with cancer: systematic review and \\ meta-analysis
}

María Teresa Rosanova ${ }^{1}$, Leticia Cuellar-Pompa. ${ }^{2}$ y Roberto Lede ${ }^{3}$

1Servicio de Control Epidemiológico e Infectología, Hospital de Pediatría Dr. Juan P. Garrahan, Bs. Aires, Argentina.
${ }^{2}$ Instituto de Investigación en Cuidados. Colegio Oficial de Enfermeros de Santa Cruz de Tenerife. España.
${ }^{3}$ Universidad Abierta Interamericana, Facultad de Medicina y Ciencias de la Salud, Argentina.

Conflictos de interés: ninguno.

Financiamiento: Ninguno.

Recibido: 20 de abril de 2021 / Aceptado: 21 de julio de 2021

\section{Resumen}

Introducción: La neutropenia febril en niños con patología oncohematològica requiere un tratamiento empírico precoz y adecuado. Esta revisión sistemática se realizó para evaluar si piperacilina/ tazobactam (PTZ) monoterapia es más efectiva y segura que los comparadores, en niños con episodios de neutropenia febril de causa oncológica. Material y Métodos: Se realizó una búsqueda bibliográfica en Embase, MEDLINE utilizando los términos de búsqueda (('febrile neutropenia' OR hemato oncology OR haemato oncology OR 'immunocompromised host' OR 'immunocompromised patient' OR 'chemotherapy-induced febrile neutropenia') AND (piperacillin OR tazobactam OR 'piperacillin plus tazobactam' OR 'piperacillin/tazobactam' OR 'piperacillin-tazobactam' OR tazocin OR 'piperacillin-tazobactam drug combination')). El criterio de valoración de eficacia fue la incidencia de fracaso terapéutico. El punto final de seguridad fue la ausencia de cualquier efecto adverso (EA). Resultados: Se identificaron 1.388 estudios, de los cuales se incluyeron 11 que cumplían los criterios de elegibilidad. Los estudios presentaron notable homogeneidad $\left(I^{2} 0 \%\right)$ y no se detectó sesgo de publicación (p 0,36). El riesgo de fracaso terapéutico de PTZ no fue mayor que en los comparadores (RR global: 0,94; IC95\% 0,83 a 1,07) como tampoco lo fue, la incidencia de EA. Conclusiones: El riesgo de fracaso terapéutico no fue superior para la PTZ como monoterapia frente a los comparadores

Palabras clave: neutropenia febril; piperacilina; tazobactan; riesgo de fracaso terapéutico.

\section{Abstract}

Background: Febrile neutropenia in children with onco-hematological diseases is an important cause of morbidity and mortality and requires early and adequate empirical treatment. This systematic review was conducted to evaluate if piperacillin/tazobactan (PTZ) monotherapy leads to a lower incidence of therapeutic failures than comparators. Methods: A literature search was carried out in Embase, and MEDLINE databases using the search terms ('febrile neutropenia' OR hemato oncology OR haemato oncology OR 'immunocompromised host' OR 'immunocompromised patient' OR 'chemotherapyinduced febrile neutropenia') AND (piperacillin OR tazobactam OR 'piperacillin plus tazobactam' OR 'piperacillin/tazobactam' OR 'piperacillin-tazobactam' OR tazocin OR 'piperacillin-tazobactam drug combination')), Efficacy endpoint was treatment failure rate. The safety end-point was absence of any adverse effects (AE). Results: Eleven studies were included. No heterogeneity was detected $\left(I^{2} 0 \%\right)$. The risk of failure was not superior for piperacillin/tazobactan to comparators (Global RR: 0.94; IC95\% 0.83 a 1.07). Rates of adverse events were similar among studies. No publication bias was detected ( $p$ 0.36). Conclusions: This systematic review and meta-analysis showed that treating episodes of febrile neutropenia in oncology pediatric patients, the risk of failure for PTZ was not superior to comparators. Adverse events were similar to the comparators.

Key words: febrile neutropenia; piperacillin; tazobactam; risk of treatment failure. 
EA asociados al tratamiento empírico de los episodios de NF con PTZ, son mayores que al ser tratados con un comparador.

as infecciones constituyen una de las primeras causas de mortalidad en pacientes con neutropenia febril (NF) secundaria a quimioterapia ${ }^{1-3}$. El tratamiento recomendado para pacientes pediátricos con cáncer y un episodio de fiebre y neutropenia catalogados de alto riesgo, incluye el ingreso hospitalario para la administración de antimicrobianos de amplio espectro por vía parenteral ${ }^{1} \mathrm{y}$, dado que se trata de una emergencia infectológica, es trascendente efectuar un correcto abordaje con el tratamiento empírico. Tanto las guías de manejo para pacientes adultos como pediátricos, señalan que el tratamiento empírico inicial en los episodios de neutropenia febril de alto riesgo (NFAR) debe incluir un antimicrobiano con cobertura contra Pseudomonas aeruginos $a^{1-5}$. Para ello, han sido propuestas como posibles opciones las cefalosporinas de tercera o de cuarta generación con acción anti-pseudomónica (ceftazidima y cefepime), los carbapenémicos (imipenem o meropenem) y las penicilinas anti-pseudomónicas (piperacilina/ tazobactam).

\section{Justificación}

Piperacilina/tazobactam (PTZ) es un fármaco compuesto por un antimicrobiano $\beta$-lactámico $(\mathrm{P})$ y un inhibidor de $\beta$-lactamasa (TZ) que es activo frente a microorganismos grampositivos, gramnegativos y anaerobios estrictos. La presencia de $\mathrm{TZ}$ en la combinación amplía el espectro antimicrobiano frente a bacterias productoras de $\beta$-lactamasas, tales como Staphylococcus aureus, Haemophilus influenzae, Bacteroides fragilis, Klebsiella spp, Escherichia coli y Acinetobacter spp. ${ }^{1,2}$.

Existe controversia si en pacientes pediátricos con NFAR, la PTZ podría ser una alternativa eficaz y segura comparada con opciones de mayor espectro, como los carbapenemes $^{1-6}$. En estudios en pacientes adultos con NF, PTZ demostró ser segura y eficaz como tratamiento, pero en pediatría, los datos aún son escasos ${ }^{6-8}$. Por lo tanto, surge la pregunta si en los pacientes pediátricos, la administración de PTZ en forma de monoterapia en esos episodios conduce a menos fracasos terapéuticos y sin mayor incidencia de efectos adversos (EA) (si es más eficaz y segura) que otras opciones de antimicrobianos en el tratamiento de episodios de NF asociadas a patologías oncológicas. Para responderla, se llevó a cabo una revisión sistemática (RS) cuali-cuantitativa de las evidencias científicas publicadas.

\section{Objetivos}

Primario: Evaluar la eficacia del tratamiento empírico con PTZ como monoterapia en los episodios de NF asociados a patologías oncológicas.

Secundario: Evaluar si la incidencia e intensidad de

\section{Material y Métodos}

En la ejecución de esta RS, se siguieron las pautas metodológicas establecidas por el acuerdo PRISMA?

\section{Búsqueda bibliográfica}

Para llevar a cabo esta revisión, se diseñaron diferentes estrategias de búsqueda para cada una de las siguientes bases de datos bibliográficas (Tabla 1): Medline (a través de PubMed), Embase (a través de Embase-Elsevier, The Cochrane Library (a través de Wiley), Cinahl (a través de EbscoHOST), SCI-EXPANDED y Scielo (a través de la WOS) y Scopus (a través de Scopus-Elsevier). La estrategia de búsqueda se probó de manera preliminar por un documentalista en la base de datos Embase. Estas primeras pruebas fueron revisadas de manera independiente por uno de los autores participantes (LCP). Una vez definida la estrategia definitiva, se tradujo a la sintaxis de las otras bases de datos seleccionadas. Se utilizaron los términos de búsqueda ('febrile neutropenia' OR hemato oncology OR haemato oncology OR 'immunocompromised host' OR 'immunocompromised patient' OR 'chemotherapyinduced febrile neutropenia') AND (piperacillin OR tazobactam OR 'piperacillin plus tazobactam' OR 'piperacillin/tazobactam' OR 'piperacillin-tazobactam' OR tazocin OR 'piperacillin-tazobactam drug combination')), con los siguientes filtros: ensayo clínico, revisión, estudio comparativo, revisiones sistemáticas, ensayo controlado aleatorizado (ECA), ensayo clínico controlado, metaanálisis y uso en estudios humanos, niños y adolescentes. Se revisaron los artículos publicados en

\begin{tabular}{|c|c|c|c|}
\hline Base de datos & $\begin{array}{c}\text { Plataforma de } \\
\text { acceso }\end{array}$ & Fecha acceso & $\begin{array}{l}\mathrm{N}^{\circ} \text { de resultados } \\
\text { obtenidos }\end{array}$ \\
\hline MEDLINE & PubMed & $24 / 02 / 2021$ & 454 \\
\hline EMBASE & Elsevier-Embase & $24 / 02 / 2021$ & 82 \\
\hline SCI-EXPANDED & WOS & $24 / 02 / 2021$ & 117 \\
\hline SCIELO & WOS & $24 / 02 / 2021$ & 2 \\
\hline SCOPUS & Elsevier & $24 / 02 / 2021$ & 652 \\
\hline CINAHL & EbscoHOST & $24 / 02 / 2021$ & 20 \\
\hline THE COCHRANE LIBRARY & Wiley & $24 / 02 / 2021$ & 62 \\
\hline Total & & & 1.388 \\
\hline Duplicados & & & 594 \\
\hline Total sin duplicados & & & 794 \\
\hline
\end{tabular}


inglés, español o francés publicados hasta febrero de 2021. Las listas de referencias de los artículos se revisaron manualmente buscando identificar estudios relevantes adicionales. De los artículos en los que no fue posible obtener el texto completo, se extrajeron los datos desde el resumen o se descartaron si los datos eran insuficientes.

\section{Medidas de Resultado}

Se adoptó a la variable fracaso terapéutico, como punto final de eficacia, basado en el concepto de que al indicar un tratamiento es de interés primordial del médico conocer el riesgo de no lograr el objetivo terapéutico, ya sea por azar, ineficacia o razones de seguridad del tratamiento indicado. Una menor incidencia de fracasos terapéuticos sugiere un mejor comportamiento del tratamiento y respalda su indicación. Los análisis de resultados se realizaron siguiendo la estrategia "según la intención de tratamiento". El punto final de seguridad se definió como la ausencia de cualquier EA obligara a la suspensión del tratamiento.

Criterios de elegibilidad de los estudios: Los estudios se incluyeron:

- Si era una investigación clínica primaria con asignación aleatorizada del tratamiento, tanto de manera experimental o cuasi experimental o abierta o cerrada y que comparara la eficacia y seguridad de la monoterapia con PTZ versus otros agentes antibacterianos en niños con cáncer y con episodios de NF.

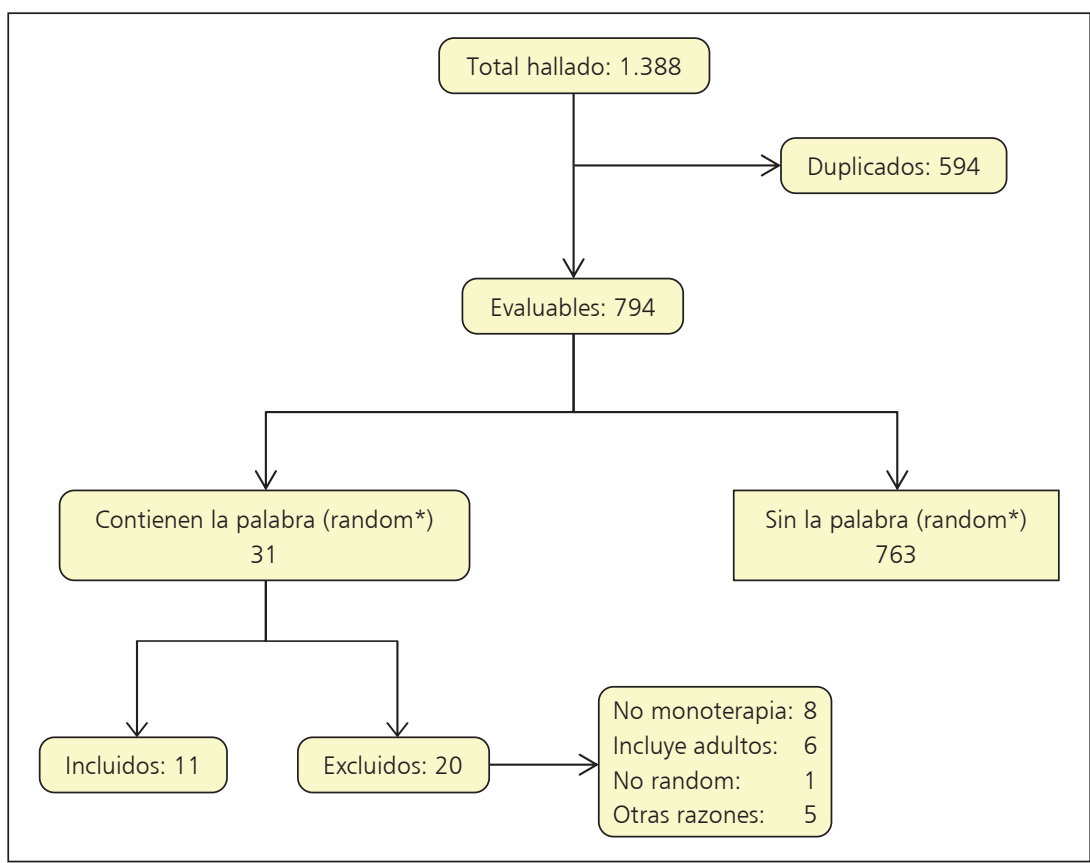

Figura 1. Flujograma de la inclusión de artículos.
- Que presentara como punto final de resultado la evolución clínica de los pacientes en el episodio de neutropenia febril, expresado como mejoría o desaparición de la fiebre sin necesidad de rotar el antimicrobiano dentro de las primeras $72 \mathrm{~h}$.

- Que no tuvieran una pérdida de pacientes $>20 \%$ de los inicialmente incluidos en cada grupo.

Dos revisores (MTR y RL) realizaron en forma independiente, la extracción de datos y la evaluación cualicuantitativa de los estudios. Los casos de desacuerdo se discutieron hasta alcanzar un consenso acorde a la validez de la evidencia.

\section{Análisis de datos y métodos estadísticos}

Se calculó el riesgo relativo (RR) individual de cada estudio y el común, con sus intervalos de confianza (IC) del 95\% para el riesgo de fracaso, utilizando el método de efecto fijo (Mantel-Haenszel) o el modelo de efectos aleatorios (Der-Simmonian-Laird), según el análisis de heterogeneidad. Los cálculos se llevaron a cabo utilizando el calculador Metaanalysis del software EPIDATA (WHO) versión 3.1. El sesgo de heterogeneidad se estimó de acuerdo a la prueba Q (Der Simonian-Laird) y se calculó el valor de $I^{2}$. El sesgo de publicación se estimó mediante la prueba de Egger. Se midió la participación porcentual de cada estudio mediante una prueba de sensibilidad. La hipótesis nula se rechazó si $\mathrm{p}<0,05$.

\section{Definiciones}

- Episodio de NF: Recuento absoluto de neutrófilos $<500$ céls $/ \mathrm{mm}^{3}$ o $<1.000$ céls $/ \mathrm{mm}^{3}$ (cuando se predice por la quimioterapia recibida un descenso de los neutrófilos a una cifra $<500$ céls $/ \mathrm{mm}^{3}$ en las 24 o 48 h siguientes) y deben presentar además una temperatura axilar $>38,5^{\circ} \mathrm{C}$ o dos mediciones $>38^{\circ} \mathrm{C}$, con una separación de al menos una hora entre una y otra.

- Eventos adversos ${ }^{10}$ : Cualquier signo desfavorable y no intencionado (incluyendo un hallazgo anormal de laboratorio), síntoma o enfermedad temporalmente asociada con el uso de PTZ o el comparador, esté o no relacionado con el medicamento administrado.

\section{Resultados}

Se detectaron 1.388 artículos de potencial interés. Una vez eliminados los duplicados, restaron 794 referencias, sobre las cuales se realizó el proceso de selección final mediante la identificación de todas aquellas que incluyeran en sus títulos o resúmenes, el término random*. La procedencia y flujo de los artículos analizados se presenta en la Tabla 1 y la Figura 1, expone la secuencia de selección, habiendo quedado 11 artículos aptos para ser incluidos en la RS. 


\section{Estudios excluidos}

Se excluyeron 20 artículos debido a diferentes motivos: ocho por no ser con monoterapia; seis por incluir adultos; uno por no asignar aleatoriamente las opciones en prueba y cinco por otras razones (comparadores no utilizados en la actualidad; comparaciones de posologías o de vías de administración).

\section{Estudios incluidos}

Finalmente, se incluyeron 11 estudios ${ }^{11-21}$ que involucraron la participación de 771 niños que aportaron 1.826 episodios febriles, de los cuales 1.796 integran la fase cuantitativa de la revisión. Los estudios incluidos no aclaraban si era un episodio de NFAR o no.

La descripción de los mismos y sus resultados individuales están disponibles en la Tabla 2. Ningún estudio presentó resultados diferentes entre los tratamientos probados, todos los IC95\% incluyeron a la unidad. La incidencia global de fracaso terapéutico entre los que recibieron PTZ, fue $34,2 \%$ y en los que recibieron el comparador, fue $35,9 \%$. No se detectó evidencia de superioridad en la incidencia de fracaso terapéutico entre PTZ y el comparador (RR global: 0,94; IC95\% 0,83 a 1,07) (Figura 2). El metaanálisis acumulativo, muestra que, al incrementarse la cantidad de pacientes en la muestra global, se redujo la incertidumbre (IC más estrechos), llegando a la actualidad en la que puede decirse que no hay duda razonable para suponer que el riesgo de fracaso terapéutico se incremente al indicar monoterapia con PTZ (Figura 3). No se detectó heterogeneidad entre los estudios incluidos (Q 9,02; $I^{2} 0 \%$ ). Este concepto se refuerza al observar la similitud del RR global calculado según los modelos fijo y aleatorio. También se analizó el cambio relativo del resultado global que se generaba por no considerar a un determinado estudio en el cálculo del resultado global. Entre ellos, se destaca el estudio de Sano y cols. ${ }^{19}$, por ser el que más elevó en RR $(1,63 \%)$ y el de Kobayashi y cols., ${ }^{21}$ por ser el que más lo redujo (-4,5\%), aunque ambos lo hacen en una proporción no relevante. Los dos estudios, compararon PTZ vs meropenem, y utilizaron la misma posología. El estudio de Sano y cols. ${ }^{19}$, es cuasi-experimental pues la asignación de los tratamientos se realizó por un método no seguro, mientras que el de

\begin{tabular}{|c|c|c|c|c|c|}
\hline \multirow[t]{2}{*}{ Autor, Año } & \multirow[t]{2}{*}{ Diseño del estudio } & \multirow{2}{*}{$\begin{array}{l}\text { N pacientes / N episodios por } \\
\text { grupo }\end{array}$} & \multicolumn{2}{|c|}{ Intervención y dosis/día } & \multirow[t]{2}{*}{ RR; IC95\% } \\
\hline & & & PTZ & Comparador & \\
\hline $\begin{array}{l}\text { Figuera, } \\
2001\end{array}$ & $\begin{array}{c}\text { Abierto. } \\
\text { Cuasi-experimental }\end{array}$ & $\begin{array}{l}137 \text { pacientes; } 69 \text { episodios con } \\
\text { PTZ y } 68 \text { en comparador }\end{array}$ & $4 / 0,5 \mathrm{~g} \mathrm{IV} \mathrm{c/} 6 \mathrm{~h}$ & $\begin{array}{c}\text { Imipenem/cilastatina, } 500 \mathrm{mg} \\
\text { IV c/6 h }\end{array}$ & $\begin{array}{c}1,18 \\
0,37 \text { a } 3,69\end{array}$ \\
\hline Corapcioglu, 2006 & $\begin{array}{c}\text { Abierto. } \\
\text { Cuasi-experimental }\end{array}$ & $\begin{array}{c}27 \text { pacientes; } 25 \text { episodios en } \\
\text { cada grupo }\end{array}$ & $\begin{array}{l}\text { Pip } 80 \text { mg/kg y Tazo } 10 \text { mg/ } \\
\mathrm{kg} \mathrm{c} / 6 \mathrm{~h} \text { (max } 4,5 \mathrm{~g} / \text { dosis) }\end{array}$ & $\begin{array}{l}\text { Cefepime, } 50 \text { mg/kg } \\
\text { c/ } 8 \text { h (max } 2 \text { g/dosis) }\end{array}$ & $\begin{array}{c}0,64 \\
0,38 \text { a } 1,08\end{array}$ \\
\hline Uygun, 2009 & $\begin{array}{c}\text { Abierto. } \\
\text { Cuasi-experimental }\end{array}$ & $\begin{array}{l}69 \text { pacientes; } 65 \text { episodios con } \\
\text { PTZ y } 62 \text { en comparador }\end{array}$ & $\begin{array}{c}80 \mathrm{mg} / \mathrm{kg} \mathrm{pip} / 10 \mathrm{mg} / \mathrm{kg} \\
\text { tazo, c/6 h }\end{array}$ & $\begin{array}{c}\text { Cefepime } \\
50 \mathrm{mg} / \mathrm{kg} \mathrm{c} / 8 \mathrm{~h}\end{array}$ & $\begin{array}{c}1,07 \\
0,69 \text { a } 1,67\end{array}$ \\
\hline Vural, 2010 & $\begin{array}{c}\text { Abierto. } \\
\text { Cuasi-experimental }\end{array}$ & $\begin{array}{l}63 \text { pacientes; } 33 \text { episodios con } \\
\text { PTZ y } 30 \text { en comparador }\end{array}$ & $360 \mathrm{mg} / \mathrm{kg} / \mathrm{d}$, en 4 dosis IV & $\begin{array}{l}\text { Imipenem-cilastatin } \\
60 \mathrm{mg} / \mathrm{kg} / \mathrm{d} \text { en } 4 \text { dosis IV }\end{array}$ & $\begin{array}{c}0,75 \\
0,47 \text { a } 1,21\end{array}$ \\
\hline Ichikawa, 2011 & $\begin{array}{c}\text { Abierto. } \\
\text { Cuasi-experimental }\end{array}$ & $\begin{array}{l}51 \text { pacientes; } 57 \text { episodios con } \\
\text { PTZ y } 62 \text { en comparador }\end{array}$ & $125 \mathrm{mg} / \mathrm{kg}$ cada $8 \mathrm{~h}$ & Cefozopran $25 \mathrm{mg} / \mathrm{kg} \mathrm{c} / 6 \mathrm{~h}$ & $\begin{array}{c}0,86 \\
0,57 \text { a } 1,30\end{array}$ \\
\hline Karaman, 2012 & $\begin{array}{c}\text { Abierto. } \\
\text { Cuasi-experimental }\end{array}$ & $\begin{array}{l}55 \text { pacientes; } 52 \text { episodios con } \\
\text { PTZ y } 50 \text { en comparador }\end{array}$ & $\begin{array}{c}360 \mathrm{mg} / \mathrm{kg} / \mathrm{d} \text {, IV en } 3 \\
\text { dosis } / \mathrm{d}\end{array}$ & $\begin{array}{c}\text { Cefoperazona/sulbactam } \\
\text { sulbactam } \\
100 \text { mg/kg/d, IV en } 3 \text { dosis/d }\end{array}$ & $\begin{array}{c}0,87 \\
0,54 \text { a } 1,39\end{array}$ \\
\hline Aamir, 2015 & $\begin{array}{l}\text { Abierto. Experimental. } \\
\text { En ramas paralelas }\end{array}$ & $\begin{array}{c}40 \text { pacientes; } 20 \text { episodios en } \\
\text { cada grupo }\end{array}$ & $100 \mathrm{mg} / \mathrm{kg} \mathrm{c} / 8 \mathrm{~h}$ & Cefepime, $50 \mathrm{mg} / \mathrm{kg} \mathrm{c} / 8 \mathrm{~h}$ & $\begin{array}{c}1,25 \\
0,39 \text { a } 3,98\end{array}$ \\
\hline Sano, 2015 & $\begin{array}{c}\text { Abierto. } \\
\text { Cuasi-experimental }\end{array}$ & $\begin{array}{l}53 \text { pacientes; } 103 \text { episodios con } \\
\text { PTZ y } 110 \text { en comparador }\end{array}$ & $337,5 \mathrm{mg} / \mathrm{kg} / \mathrm{d}$ & Cefepime $100 \mathrm{mg} / \mathrm{kg} / \mathrm{d}$ & $\begin{array}{c}1,16 \\
0,85 \text { a } 1,57\end{array}$ \\
\hline Sano, 2016 & $\begin{array}{c}\text { Abierto. } \\
\text { Cuasi-experimental }\end{array}$ & $\begin{array}{l}105 \text { pacientes; } 226 \text { episodios con } \\
\text { PTZ y } 208 \text { en comparador }\end{array}$ & $\begin{array}{c}337,5 \mathrm{mg} / \mathrm{kg} / \mathrm{d} \text {, en } 3 \text { dosis. } \\
\text { Max/día 13,5 g }\end{array}$ & $\begin{array}{l}\text { Meropenem } 120 \mathrm{mg} / \mathrm{kg} / \mathrm{d} \text { en } \\
3 \text { dosis. Max/día } 3 \mathrm{~g}\end{array}$ & $\begin{array}{c}1,10 \\
0,85 \text { a } 1,41\end{array}$ \\
\hline Kamorattana, 2019 & $\begin{array}{l}\text { Abierto. Experimental. } \\
\text { En ramas paralelas }\end{array}$ & $\begin{array}{c}70 \text { pacientes; } 59 \text { episodios en } \\
\text { cada grupo }\end{array}$ & $\begin{array}{c}320 \mathrm{mg} / \mathrm{kg} / \text { day dividido en } \\
\text { dosis } \mathrm{c} / 8 \mathrm{~h}\end{array}$ & $\begin{array}{l}\text { Ceftazidima } 100 \mathrm{mg} / \mathrm{kg} / \mathrm{d} \\
\text { dividida } \mathrm{c} / 8 \mathrm{~h}+\text { amikacina } \\
15 \mathrm{mg} / \mathrm{kg} / \mathrm{d} \text { en una dosis } / \mathrm{d}\end{array}$ & $\begin{array}{c}0,69 \\
0,42 \text { a } 1,12\end{array}$ \\
\hline Kobayashi, 2020 & $\begin{array}{l}\text { Abierto. Experimental. } \\
\text { En ramas paralelas }\end{array}$ & $\begin{array}{l}99 \text { pacientes; } 193 \text { episodios con } \\
\text { PTZ y } 200 \text { en comparador }\end{array}$ & $\begin{array}{c}360 \mathrm{mg} / \mathrm{kg} / \mathrm{d} \text { IV en } 1 \mathrm{~h}, \\
\mathrm{c} / 6 \mathrm{~h}\end{array}$ & $\begin{array}{c}\text { Meropenem } 120 \mathrm{mg} / \mathrm{kg} / \mathrm{d} \text { IV en } \\
1 \mathrm{~h}, 3 \text { veces al día }\end{array}$ & $\begin{array}{c}0,86 \\
0,63 \text { a } 1,18\end{array}$ \\
\hline
\end{tabular}




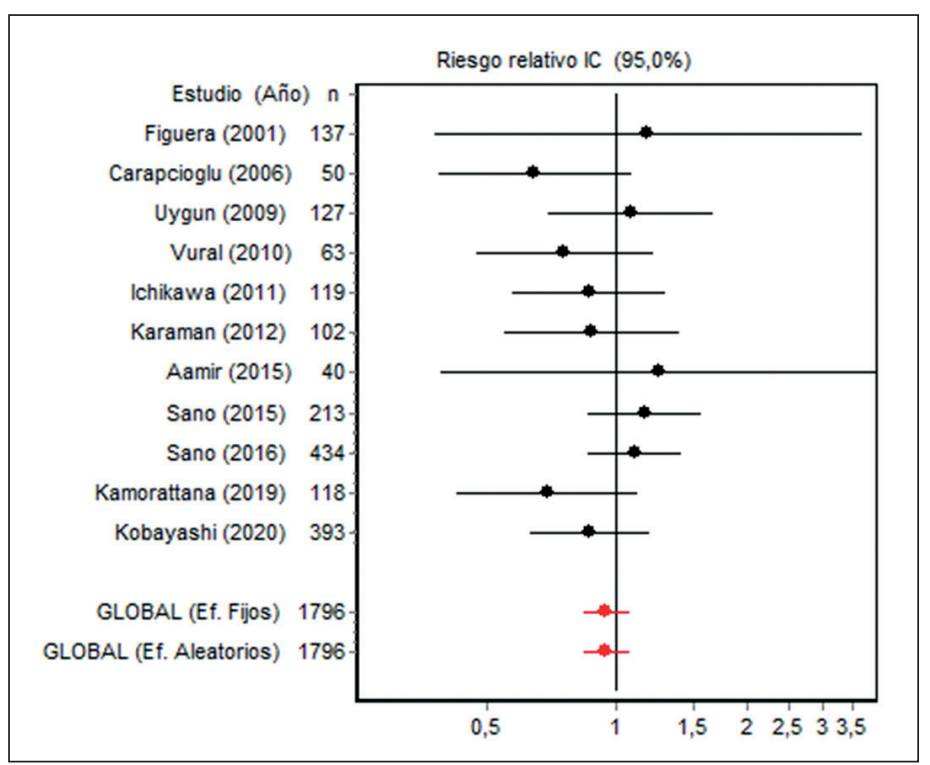

Figura 2. Fracaso terapéutico de piperacilina/tazobactam en episodios de neutropenia febril vs comparador. Gráfico de resultados ("forest plot").

Kobayashi y cols. ${ }^{21}$, es experimental, pues la asigna mediante sobres cerrados. Dadas las similitudes entre ambos estudios puede admitirse que la variación es solamente azarosa.

Las referencias a los EA no están expuestas de manera sistemática entre los estudios, pero se aprecia que la incidencia es baja, predominando los EA leves tales como "rash" o diarrea que no obligaron a la suspensión del tratamiento. Sano y cols. ${ }^{18}$, refieren una muerte en el grupo PTZ en un paciente con microbiología negativa y en su estudio de $2017^{19}$ refiere dos muertes en pacientes infectados en el mismo grupo de tratamiento, aunque no las relaciona al mismo. Aamir y cols. ${ }^{17}$, informan cuatro muertes en el grupo PTZ y dos en el comparador, pero esa diferencia no es estadísticamente significativa ni aclara si esos casos fueron incluidos en los análisis, como tampoco lo hacen Figuera y cols., ${ }^{11}$ que también refiere decesos. Este autor, informa mayor incidencia de vómitos entre los que recibían el comparador (imipinem, $39,7 \%$ vs 5,6\%; $<<0,0001)$. La proporción de episodios febriles con certificación microbiológica informada fue $<50 \%$ y se constató que la exposición de los resultados fue irregular, lo que no permitió establecer comparaciones útiles entre los trabajos.

No se detectó sesgo de publicación (p 0,36) (Figura 4).

\section{Discusión}

La NF constituye una importante causa de morbi-mortalidad en niños con cáncer ${ }^{1}$. El tratamiento empírico inicial (TEI) debe tener un amplio espectro, ser bactericida y deben considerarse los microorganismos predominantes en cada centro asistencial ${ }^{1-6}$.

Por muchos años, la terapia antimicrobiana combinada con aminoglucósidos fue considerada de elección en el TEI ${ }^{3-6}$. Sin embargo, algunos trabajos han demostrado que la monoterapia podría ser

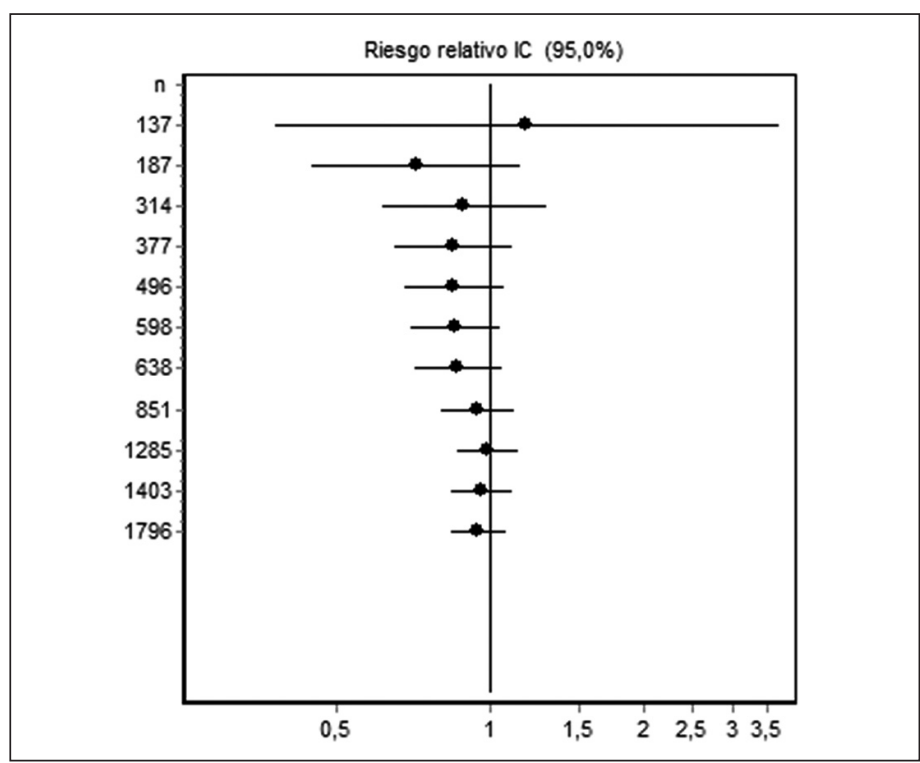

Figura 3. Riesgo de fracaso terapéutico en el tratamiento de episodios de neutropenia febril con piperacilina/tazobactam. Metaanálisis acumulativo.

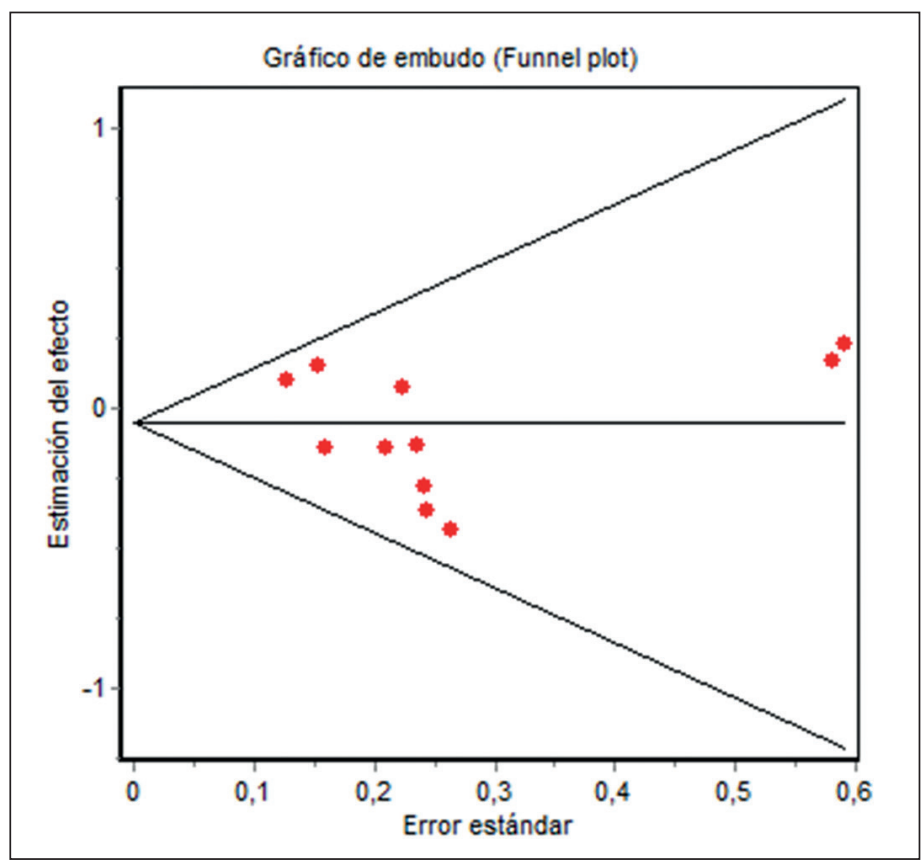

Figura 4. Evaluación del sesgo de publicación en ensayos terapéuticos con piperacilina/ tazobactam en episodios de neutropenia febril.

igualmente segura y eficaz y en la actualidad, se tiende a reemplazar al tratamiento empirico inicial combinado en pacientes con NF de causa oncohematológica. Los carbapenemes son una opción de monoterapia para el tratamiento empírico inicial en pacientes con NFAR, pero su uso de manera indiscriminada puede favorecer el incremento de bacterias productoras de carbapenemasas, lo que ha 
todos los $\beta$-lactámicos con actividad anti $P$. aeruginosa, concluyen que tanto PTZ como meropenem o la asociación imipenem/cilastina, son una opción razonable como tratamiento de primera elección. Paul y cols., también citado en el trabajo de Horita y cols. ${ }^{22-23}$, concluyen que la mortalidad fue menor con PTZ en el TEI de pacientes con NF. Ambos estudios incluyen trabajos experimentales y no experimentales, realizados en población sin restricción de edad y las conclusiones de ambos son coincidentes con la del presente estudio. En estos trabajos, la PTZ tuvo menores EA que los carbapenemes.

Destacamos que la fortaleza de este estudio reside en una búsqueda bibliográfica exhaustiva y la ausencia de heterogeneidad entre los diferentes estudios incluidos, lo que avala la solidez de la conclusión.

De lo anterior, se deduce que la respuesta a la pregunta clínica inicial, de clara importancia para la práctica asistencial, es que la administración de PTZ en forma de monoterapia como TEI de los episodios de NF asociados a patología oncológica en pacientes pediátricos, no conduce a menos fracasos terapéuticos que si se indican otras opciones de antimicrobianos de mayor espectro, como los carbapenemes.

Una debilidad es que en los trabajos incluidos no se encontró la categorización de riesgo de los episodios de NF y no se informaron adecuadamente los hallazgos microbiológicos de los mismos. La definición, al ingreso del paciente, de estar cursando un episodio de NF con bajo $o$ alto riesgo de tener una infección bacteriana, permitiría abstenerse de emplear una terapia anti-pseudomonas como la evaluada en la presente RS en aquellos episodios de bajo riesgo. Entendemos que es conveniente la categorización inicial del riesgo y que los patrones de resistencia locales sean considerados en cada centro antes de indicar un tratamiento empírico y de extrapolar estas conclusiones.

\section{Referencias bibliográficas}

1.- Lehrnbecher T, Robinson P, Fisher B, Alexander S, Ammann R A, Beauchemin $\mathrm{M}$, et al. Guideline for the management of fever and neutropenia in children with cancer and hematopoietic stem-cell transplantation recipients: 2017 update. J Clin Oncol 2017; 35: 2082-94. doi: 10.1200/JCO.2016.71.7017.

2.- Pacheco-Rosas D O, Peregrino-Bejarano L, López-Aguilar J E, Juan-Shum L, et al. Piperacilina/tazobactam más amikacina vs. piperacilina/tazobactam: tratamiento en niños con neutropenia febril. Rev Med Inst Mex Seguro Soc 2019; 57: 65-73. PMID: 31617992.

3.- Demirel A, Tabak F, Ar M C, Mete B, Mete B, Öngören S, et al. Secondary infections in febrile neutropenia in hematological malignances: more than another febrile neutropenic episode. Turk J Haematol 2015; 32: 243-50. doi: 10.4274/tjh.2013.0422.

4.- Aguilera-Alonso D, Escosa-García L, Goycochea-Valdivia A, Soler-Palacínd, Saavedra-Lozano J, Rodrigo C, et al. Documento de posicionamiento de la Asociación Española de Pediatría-Sociedad Española de Infectología Pediátrica (AEP-SEIP) sobre el tratamiento de las infecciones por bacterias multi-resistentes. An Pediatr (Barc) 2019; 91: 351. e1-351.e13. doi: 10.1016/j.anpedi.2019.08.002.

5.- Lehrnbecher T. Treatment of fever in neutropenia in pediatric oncology patients. Curr Opin Pediatr 2019; 31: 35-40. doi: 10.1097/ MOP.0000000000000708.

6.- Sano H, Kobayashi R, Suzuki D, Kishimoto
K, Hori D, Matsushima S, et al. Differential efficacy of empirical antibiotic therapy for febrile neutropenia in adolescent/young adult (AYA) and child patients. Int J Hematol 2018; 108: 543-9. doi: 10.1007/s12185-018-2503-6.

7.- Demirkaya M, Celebi S, Sevinir B, Hacimustafaoglu M. Randomized comparison of piperacillin-tazobactam plus amikacin versus cefoperazone/sulbactam plus amikacin Pediatr Hematol Oncol 2013; 30: 141-8. doi: 10.3109/08880018.2012.756565.

8.- Scheler M, Lehrnbecher T, Groll A H, Volland R, LawsH J, Ammann R A, et al. Management of children with fever and neutropenia: results of a survey in 51 pediatric cancer centers in Germany, Austria, and Switzerland. Infection 2020; 48: 607-18. doi: 10.1007/s15010-02001462-z. 
9.- Moher D, Liberati A, Tetzlaff J, Altman D G and PRISMA Group. Preferred reporting items for systematic reviews and meta-analyses: the PRISMA statement. PLoS Med 6(7): e1000097. doi:10.1371/ journal.pmed.100009.

10.- E5 (R1) Guideline for Good Clinical Practice. https://ichgcp.net/storage/pdf/ich-gcp-en.pdf (accedido 3 de marzo de 2021)

11.- Figuera A, Rivero N, Pajuelo F, Font P, Leyra F, de La Cámara R, et al. Estudio comparativo de piperacilina/ tazobactam frente a imipenem/ cilastatina en la neutropenia febril (19941996). Med Clin (Barc) 2001; 116: 610-1. doi: 10.1016/s0025-7753(01)71921-5.

12.- Corapcioglu F, Sarper N, Zengin E. Monotherapy with piperacillin/tazobactam versus cefepime as empirical therapy for febrile neutropenia in pediatric cancer patients: a randomized comparison. Pediatric Hematol Oncol 2006; 23: 177-86. PMID: 16517534.

13.- Uygun V, Karasu G, Ogunc D, Yesilipek A, Hazar V. Piperacillin/tazobactam versus cefepime for the empirical treatment of pediatric cancer patients with neutropenia and fever: a randomized and open-label study. Pediatr Blood Cancer 2009; 53: 610-4. doi: $10.1002 / p b c .22100$.

14.- Vural S, Erdem E, Gulec S, Yildirmak Y, Kebudi R. Imipenem-cilastatin versus piperacillin-tazobactam as monotherapy in febrile neutropenia. Pediatrics Int 2010: 52; 262-7. doi: 10.1111/j.1442-200X.2009.02952.x.

15.- Ichikawa M, Suzuki D, Ohshima J, Cho Y, Makoto Kaneda, Akihiro Iguch et al.
Piperacillin/tazobactam versus cefozopran for the empirical treatment of pediatric cancer patients with febrile neutropenia. Pediatr Blood Cancer 2011; 57: 1159-62. doi: 10.1002/ pbc.23106.

16.- Karaman S, Vural S, Yildirmak Y, Emecen M, Erdem E, Kebudi R. Comparison of piperacillin tazobactam and cefoperazone sulbactam monotherapy in treatment of febrile neutropenia. Pediatr Blood Cancer 2012; 58: 579-83. doi 10.1002/pbc.23245.

17.- Aamir M, Abrol P, Sharma D, Punia H. A clinical evaluation of efficacy and safety of cefepime monotherapy versus piperacillintazobactam in patients of paediatric age group with febrile neutropenia in a tertiary care centre of north India. Trop Doct 2015; 46: 142-8. doi: $10.1177 / 0049475515617571$.

18.- Sano H, Kobayashi R, Suzuki D, Kishimoto K, Yasuda K, Kunihiko Kobayashi K. Comparison between piperacillin/tazobactam and cefepime monotherapies as an empirical therapy for febrile neutropenia in children with hematological and malignant disorders: a prospective, randomized study. Pediatr Blood Cancer 2015; 62: 356-8. doi: 10.1002/ pbc. 25178 .

19.- Sano H, Kobayashi R, Suzuki D, Hori D, Kishimoto K, Kobayashi K. A prospective randomized trial comparing piperacillin/tazobactam with meropenem as empirical antibiotic treatment of febrile neutropenic children and adolescents with hematologic and malignant disorders. Pediatr
Blood Cancer 2017; 64 (6): e26360. doi: 10.1002/pbc.26360.

20.- Kamonrattana R, Sathitsamitphong L, Choeyprasert W, Charoenkwan P, et al. A randomized, open-labeled, prospective controlled study to assess the efficacy of frontline empirical intravenous piperacillin/tazobactam monotherapy in comparison with ceftazidime plus amikacin for febrile neutropenia in pediatric oncology patients. Asian Pac J Cancer Prev 2019; 20: 2733-7. doi: 10.31557/ APJCP.2019.20.9.2733.

21.- Kobayashi R, Sano H, Matsushima S, Hori D, Masato Yanagi M, Kodama K, et al. Meropenem versus piperacillin/tazobactam for febrile neutropenia in pediatric patients: efficacy of piperacillin/tazobactam as a $1 \mathrm{~h}$ drop infusion four times a day. Intern J Hematol 2020; 113(3): 430-5. doi: 10.1007/s12185-02003031-4.

22.- Horita N, Shibata Y, Watanabe H, Namkoong $\mathrm{H}$, KaneKo T. Comparison of antipseudomonal $\beta$-lactams for febrile neutropenia empiric therapy: systematic review and network metaanalysis. Clin Microbiol Infect 2017; 23: 723-9. doi: 10.1016/j.cmi.2017.03.024.

23.- Paul M, Yahav D, Bivas A, Fraser A, Leibovici L. Anti-pseudomonal beta-lactams for the initial, empirical, treatment of febrile neutropenia: comparison of beta-lactams. Cochrane Database Syst Rev. 2010; 10 (11): CD005197. doi: 10.1002/14651858.CD005197. pub3. 